\title{
S Research Square

\section{Prognostic value of the site of distant metastasis and of surgical interventions in metastatic gastric cancer: a population-based study}

\section{Yinghua Li}

Southwest Medical University https://orcid.org/0000-0001-6133-5347

Danna Xie

Southwest Medical University

\section{Xiaojing Chen}

Southwest Medical University

\section{Teng Hu}

Southwest Medical University

\section{Simin Lu}

Southwest Medical University

\section{Yunwei Han ( $\square$ lanpaoxiansheng@126.com )}

Southwest Medical University https://orcid.org/0000-0002-0942-9095

\section{Research}

Keywords: Stomach Neoplasms, Distant metastatic site, Surgery, Prognosis

Posted Date: March 10th, 2020

DOI: https://doi.org/10.21203/rs.3.rs-16606/v1

License: (c) (1) This work is licensed under a Creative Commons Attribution 4.0 International License. Read Full License

Version of Record: A version of this preprint was published at Technology in Cancer Research \& Treatment on January 1st, 2020. See the published version at https://doi.org/10.1177/1533033820964131. 


\section{Abstract}

Background: The studies on the prognostic significance of site-specific distant metastasis, multiple-site metastases, and the impact of surgery of the primary tumor and metastatic lesion on survival outcomes of patients with metastatic gastric cancer (GC) remain elusive. Therefore, the present study aimed to investigate the prognostic significance of the site of distant metastasis among patients with metastatic GC. Furthermore, the effect of surgery of the primary tumor and metastatic lesion on the prognosis of metastatic GC was also analyzed.

Methods: The data of 4,221 eligible patients, who were diagnosed with metastatic GC between 2010 and 2015, were identified from the Surveillance Epidemiology and End Results (SEER) database. Multivariate logistic regression analysis was performed to assess the association between potential prognostic factors, including the site of metastasis and surgery, and survival of patients with metastatic GC. Overall survival (OS) and cause-specific survival (CSS) were determined using the Kaplan-Meier survival curves and differences were assessed using the Log-rank test.

Results: A total of 4,221 eligible GC patients with definite organ metastases were identified. Of these, 3312 GC patients had single-site metastasis while 909 patients had multiple-site metastases. GC Patients with single-site metastasis of liver or lung exhibited better CSS and OS compared to GC patients with bone metastasis. Furthermore, GC patients with liver metastasis were benefitted from surgery of both the primary and metastatic lesions, while GC patients with lung metastasis were benefited from surgery of metastasis resection only. Multivariate Cox regression analysis revealed that GC patients with single-site metastasis, well-differentiated tumors, and GC patients who underwent surgery of the primary tumor and those who received chemotherapy exhibited favorable prognosis.

Conclusions: The site of metastasis was an independent prognostic factor for metastatic GC. Surgery had survival benefits in certain cases of metastatic GC; however, further studies are warranted to clarify these benefits in carefully selected patients.

\section{Background}

Gastric cancer (GC) ranks the fifth most frequent malignancy diagnosed worldwide. Due to its increased tendency to metastasize to vital organs including liver, lung, bone, and brain, GC continues to be the third leading cause of cancer-specific [1]. In the United States, approximately 27,510 new cases and 11,140 deaths were estimated by the American Cancer Society in 2019[2]. Notably, the prognosis of GC depends largely on the clinical stage at presentation [3]. However, due to the lack of early alarming symptoms and limited availability of early screening programs, GC remains unnoticed until advanced stages and approximately $40 \%$ of GC patients will present with distant metastases at the time of initial diagnosis, where treatment options are limited[4]. Consequently, the prognosis of patients with metastatic GC remains poor with median survival being less than 1 year and the 5-year survival rate being less than 10\% without surgery. Nevertheless, surgery as the standard curative therapy has markedly improved the 
survival of patients with early GC; besides, a multimodality treatment strategy including surgery combined with neoadjuvant and adjuvant therapies has been recommended to improve the survival of locally advanced stages $[5,6]$. Thus, surgery represents a curative opportunity for patients with GC and is considered the foundation of multimodal management of GC [7]. In this context, several retrospective studies have provided evidence of a potential benefit of gastrectomy in metastatic GC as well [8-14].

Although chemotherapy or chemotherapy combined with targeted therapy remains the cornerstone of the palliative treatment of advanced or metastatic GC, surgery is beneficial to the survival of patients with metastatic GC. Accumulating studies have revealed that liver resection in GC patients with liver metastasis is considered favorable with marked 5-year survival and could be offered to selected patients [8]. While improved surgical interventions and adjuvant therapies remain the upfront research topic, studies on careful patient selection remain scares. Moreover, due to the paucity of epidemiological studies, predicting the prognosis of the patients with metastatic GC remains elusive [15]. Besides, the decision to perform surgery of primary tumors and/or metastases remains clinically challenging. Therefore, it becomes imperative to identify patterns of metastases in order to articulate the best treatment decision and optimize the follow-up strategies. Considering these pieces of evidence, the present study aimed to determine the epidemiology of preferential sites of distant metastasis in patients with metastatic GC. Furthermore, the effects of surgical resection of the primary tumor and metastatic lesion on the prognosis of metastatic GC were also analyzed.

\section{Methods}

\section{Data collection}

For the present study, the data of metastatic GC patients were retrieved from the Surveillance Epidemiology and End Results (SEER) database of the USA National Cancer Institute, which is the largest publicly available cancer database and consists of a consortium of 18 population-based regional cancer registries with accurate and consistent data and includes representative cancer statistics from an estimated $28 \%$ of the American population, The SEER registries routinely collect data on patients' demographic information and clinicopathological characteristics including the primary tumor site, morphology, and stage at diagnosis, first course of treatment, and follow-up for vital status. We used the SEER ${ }^{\star}$ Stat software version 8.3.6 with the approval from the SEER program (Username: 10053-Nov2018) to assess the data. The SEER database provided patients data up to 2016 based on the November 2018 submission. This study was approved by the Institutional Review Board of the Affiliated Hospital of Southwest Medical University. As this study was based on the public data of the SEER database, the requirement for informed patient consent was waived.

\section{Patient selection}

A total of 40,728 patients with GC were identified within the SEER database between 2010 and 2015. Data on the peritoneum and other uncommon metastatic organs such as adrenal glands and spleen was unavailable. Based on the inclusion and exclusion criteria, a total of 4,221 gastric adenocarcinomas with 
defined liver, lung, bone, and brain metastases were included in the study. The age at diagnosis, gender, ethnicity, primary tumor site, grade, histological type, tumor staging was determined according to the American Joint Committee on Cancer (AJCC) 7th edition staging system using available clinical and pathologic data on tumor invasion, lymph nodes status, and distant metastasis, marital status, surgery of the primary tumor, surgery of the metastases, chemotherapy, radiotherapy, survival months, and cause of death for patients with GC were collected from the SEER database. The inclusion criteria were as follows: The primary site code represented "stomach" (C16.0-16.9); histology codes denoted "adenocarcinoma" (ICD-03, 8140/3, 8142/3, 8143/3, 8144/3, 8145/3, 8210/3, 8211/3, 8255/3, 8260/3, 8261/3, 8263/3, 8310/3, 8323/3), "mucinous" (ICD-03, 8480/3, 8481/3) and "signet ring cell carcinoma" (ICD-03, 8490/3); patients with identified bone, brain, lung, or liver metastasis. The exclusion criteria were as follows: patients who survived less than 1 month; patients with incomplete data about distant metastatic sites; patients with incomplete data about primary and/or metastatic surgery; patients with missing or incomplete information about survival, follow-up months, cause of death or other characteristics. The unknown clinical data were presented as an unknown category. The data extraction process is illustrated in Fig. 1.

\section{Statistical analysis}

Cause-specific survival (CSS) and overall survival (OS) were defined as two primary endpoints of the present study. The cause-specific survival was defined as the survival time from the time of diagnosis to the date of the death from GC. The patients who died from causes unrelated to GC or who survived after the follow-up deadline was defined as censored. The overall survival was calculated from the date of diagnosis to the date of death from any cause or the last follow-up. In this study, CSS estimated the probability of surviving GC. Survival curves were estimated using the Kaplan-Meier method, and the significant differences between the survival curves were assessed by the Log-rank test.

Independent variables were first analyzed using univariate analysis. Variables that were significantly associated with surgery of primary and metastatic lesions in GC patients identified by univariate analysis were then entered into a Cox proportional hazards regression model for multivariate analysis, yielding hazard ratios (HR). The model analyzed nine significant variables, including age, gender, primary tumor site, marital status, race, histological type, TNM staging, metastatic site, and chemotherapy. All statistical analyses were two-sided. P-values of $<0.05$ were considered statistically significant. All statistical analyses were performed with R software (version 3.6.0).

\section{Results}

\section{Patient Characteristics}

A total of 4,221 eligible GC patients with definite organ metastases (liver, lung, bone, brain) were identified and included in the present study based on the inclusion and exclusion criteria. Of these, 3312 patients had single-site metastasis while 909 patients presented multiple-site metastases. In the overall patient cohort, there were 3133, 1041, 956, and 156 patients with liver, lung, bone, and brain metastases, 
respectively. Notably, the most common metastasis of single-site was liver $(70.5 \%)$, followed by bone (15.8\%), lung (11.9\%), and brain (1.8\%). The median age at diagnosis was 65 years. The clinicopathological characteristics of patients with metastatic GC are summarized in Table 1. A total of 457 patients underwent surgical resection, of these, surgical resection for primary GC resection was performed in 303 patients and in $218 \mathrm{GC}$ patients the resection of metastasis was performed. In the subgroup of patients with primary tumor resection, the percent of patients with bone metastasis was the lowest, approximately $4.0 \%$. While in the subgroup of patients with metastatic tumor resection, the percent of patients with liver metastasis was found to be the lowest, approximately $3.7 \%$. Furthermore, a total of 2796 patients received chemotherapy and 972 patients were administered with radiotherapy.

\section{Survival outcomes}

OS and CSS in patients with advanced GC were evaluated based on single-site or multiple-site metastases and surgeries performed on the primary or metastatic tumors. The results indicated that advanced GC patients with single-site bone metastasis ( $p=0.03$ for CSS), brain metastasis ( $p=0.03$ for CSS), or liver metastasis ( $p<0.001$ for CSS) were benefited the most from primary surgery $(p<0.05)$ (Fig. 2). Furthermore, the evaluation of metastatic GC patients undergoing resection for metastatic lesions revealed that patients with lung metastasis ( $p=0.007$ for CSS) and liver metastasis ( $p=0.02$ for CSS) were benefitted significantly from surgery than patients with bone metastasis and brain metastasis (Fig. 3). However, there was no significant difference between patients who underwent resection for primary and metastatic lesions in GC patients with multiple-site metastases (Supplementary Fig. 1).

In the cohort of patients who received chemotherapy, advanced GC patients with the brain $(p=0.02$ for CSS) or liver ( $p<0.001$ for CSS) metastasis were benefitted significantly from surgery of the primary tumor (Supplementary Fig. 2). However, patients with lung ( $p=0.01$ for CSS) or liver ( $p=0.02$ for CSS) metastasis benefitted significantly from surgery of the metastases (Supplementary Fig. 3).

The median OS for advanced GC patients with single-site bone, brain, lung, and liver metastases were 5, 4,6 , and 6 months, respectively. Advanced GC Patients with isolated lung or liver metastasis exhibited better CSS and OS compared with GC patients with bone metastasis ( $p=0.001$ for CSS and $p=0.002$ for OS in lung metastasis; $p=0.002$ for both CSS and OS in liver metastasis). However, no significant difference was observed between patients with single-site lung and liver metastases ( $p>0.05$ for both CSS and OS). There was no significant difference between patients with single-site bone and brain metastases. ( $p>0.05$ for both CSS and OS) (Fig. 4).

Furthermore, the prognostic value of the pattern of metastases was also evaluated for stage IV GC patients. The median OS for patients with one, two, three, and four metastatic sites was $6,4,4$, and 3 months, respectively. Patients with single-site metastasis exhibited survival benefits over patients with multiple-site metastases ( $p<0.001$ for both CSS and OS). However, no significant difference was observed between patients with two, three or four metastatic sites ( $p>0.05$ for both CCS and OS) (Supplementary Fig. 4). 


\section{Analysis of prognostic factors using Multivariable Cox regression}

Multivariate Cox regression analysis was performed to evaluate the association of significant variables with the prognosis of metastatic GC. The results indicated that both CSS and OS were significantly improved in GC patients who underwent primary resection ( $\mathrm{HR}, 0.48 ; 95 \% \mathrm{Cl}, 0.41-0.56 ; \mathrm{p}<0.001)$ and those who received chemotherapy ( $\mathrm{HR}, 0.37 ; 95 \% \mathrm{Cl}, 0.34-0.40 ; p<0.001)$. Lung metastasis (HR, $0.77 ; 95 \%$ $\mathrm{Cl}, 0.67-0.89 ; \mathrm{p}<0.001)$ and liver metastasis $(\mathrm{HR}, 0.82 ; 95 \% \mathrm{Cl}, 0.74-0.92 ; \mathrm{p}=0.001)$ was significantly associated with improved CSS and OS of GC patients compared with bone metastasis (Table 2). In the overall cohort, patients aged $>65$, undifferentiated cancer, signet ring cancer, who were single, or had multiple-site metastases were found to be statistically significant independent prognostic factors for poor survival, while primary surgical treatment and chemotherapy were protective factors. However, in the single-site metastasis cohort and the overall patient cohort, there was no significant association between the GC patients who underwent metastatic surgery and CSS/OS. (Supplementary Table 1)

\section{Prognostic factors associated with surgery}

In a multivariable logistic regression model, patients with gastric cardia cancer (OR, 3.36; $95 \% \mathrm{Cl}, 2.37$ 4.83; $p<0.001)$, who were unmarried (OR, 1.75; 95\% Cl, 1.27-2. 42; $p<0.001)$, had signet ring cell cancer $(\mathrm{OR}, 1.82 ; 95 \% \mathrm{Cl}, 1.12-3.04 ; \mathrm{p}=0.018)$, or who underwent chemotherapy (OR, 1.59; $95 \% \mathrm{Cl}, 1.17-2.16 ; \mathrm{p}=$ 0.003 ) were less likely to undergo primary surgery. Considering bone metastasis as a reference, GC patients with brain $(\mathrm{OR}, 0.20 ; 95 \% \mathrm{Cl}, 0.07-0.58 ; \mathrm{p}=0.002)$, lung $(\mathrm{OR}, 0.48 ; 95 \% \mathrm{Cl}, 0.25-0.91 ; \mathrm{p}=0.025)$ or

liver (OR, $0.52 ; 95 \% \mathrm{Cl}, 0.30-0.86 ; \mathrm{p}=0.015)$ metastasis were more likely to receive primary tumor resection (Table 3). In the metastatic tumor resection cohort, GC patients older than 65 years $(\mathrm{OR}, 1.44 ; 95 \% \mathrm{Cl}$, $1.03-2.02 ; p=0.036)$ or male patients $(O R, 1.61 ; 95 \% \mathrm{Cl}, 1.35-2.27 ; p=0.007)$ were less likely to receive metastatic tumor resection. In addition, GC patients with mucinous adenocarcinoma of the stomach (OR, $0.27 ; 95 \% \mathrm{Cl}, 0.13-0.61 ; \mathrm{p}<0.001)$ and $\mathrm{GC}$ patients who underwent primary resection $(\mathrm{OR}, 0.13 ; 95 \% \mathrm{Cl}$, $0.08-0.19 ; p<0.001$ ) were more likely to receive metastasis resection. Compared to GC patients with liver metastasis, $\mathrm{GC}$ patients with brain metastasis (OR, 0.07; 95\% Cl, 0.04-0.14; $\mathrm{p}<0.001)$, lung metastasis (OR, $0.37 ; 95 \% \mathrm{Cl}, 0.24-0.58 ; \mathrm{p}<0.001$ ) or bone metastasis (OR, $0.44 ; 95 \% \mathrm{Cl}, 0.28-0.69 ; \mathrm{p}<0.001$ ) were more likely to undergo metastatic surgery (Supplementary Table 2).

\section{Discussion}

Although metastatic GC is recognized as the terminal stage, carefully selected patients may exhibit an improved prognosis following primary tumor resection surgery or surgery to remove metastases [11]. Besides, the sites of metastasis and the pattern of metastatic sites can impact the prognosis of metastatic GC. The poor prognosis of metastatic GC is often attributed to the involvement of the liver, lung, brain and, bone metastases. Therefore, a better understanding of the epidemiology of metastatic GC remains highly desirable to actively investigate and articulate new treatment strategies [16]. In this study, we found that the prognosis of patients with liver or lung metastasis was significantly better than that of patients with bone metastasis. Conceivably, GC patients with brain or bone metastasis will be 
significantly benefitted from primary resection, while metastatic resection might prove to be highly beneficial to GC patients with lung metastasis.

In the present study, the metastatic rate to liver, lung, bone and brain metastases in patients with GC was $16.0 \%, 5.3 \%, 4.9 \%$, and $0.8 \%$, respectively. Consistent with previous reports, the results of the present study also indicated that GC most commonly metastasized to the liver through direct invasion and hematogenous metastasis and that GC frequently metastasizes first to the liver [17-22]. Besides, owing to the increased availability of abdominal CT and ultrasound, liver metastasis is detected in a large proportion of metastatic GC. Furthermore, the proportion of patients with isolated bone metastasis was significantly higher than that of patients with isolated lung metastasis. Similarly, Qiu et al. also reported that the number of GC patients with bone metastases was comparable to that of lung metastases [23]. Apparently, advanced bone metastases are most often established when the patient complains of bone pain or suffers a pathological fracture; consequently, early asymptomatic bone metastases remain unnoticed. With the increasing acceptance of bone scans, it is likely that more patients will be diagnosed in the early asymptomatic phase of bone metastasis [24].

Using a multivariate logistic regression model, the present study revealed that a number of variables affect the surgical planning of metastatic GC. As the median age of the study population was 65 years, we selected 65 years as the cutoff point. The low resection rate was detected in single patients or those living alone which may be attributed to the lack of financial and emotional support from their family. Similarly, Liu et al. also reported that being single or living alone was associated with poor surgical rates [25].

Histopathologically, signet ring cell carcinoma is more aggressive and often exhibit poor surgical compliance [26]. Due to the low resection rate of the primary tumor in the GC patients with bone metastasis, we used bone metastasis as a reference to analyze the effects of other metastasis on primary tumor resection. Likewise, we analyzed the effect of other metastatic sites on the metastatic lesion resection due to the low rate of liver resection. We found that patients who had undergone primary tumor resection were more likely to have metastases removed. Of 303 patients who received primary lesion resection, 64 patients also underwent metastatic lesion resection. Although chemotherapy alone cannot improve the survival rate of advanced GC, a few studies have suggested that chemotherapy can markedly support the resection of primary or metastatic tumors, thus improving the prognosis [27]. In this study, we found that a reduction in patients undergoing primary tumor resection following neoadjuvant chemotherapy as the initial treatment. Thus, we assumed that this might be related to the transient tumor-shrinking effect of chemotherapy.

Notably, we also found that nearly $10 \%$ of patients with metastatic GC received surgical treatment. This might be attributed to the fact that the majority of patients had surgical intolerance or a lack of awareness. Furthermore, this study revealed that patients with isolated liver metastasis exhibited an improved prognosis following resection of the primary and metastatic lesions. Consistent with a study by Cheon et al., hepatectomy would be beneficial for GC patients with liver metastases [28]. Some studies 
have suggested that GC patients with isolated lung metastasis have a better survival prognosis compared with GC patients with isolated liver metastasis[29]. Conversely, there was no significant difference in survival benefit between GC patients with liver metastasis and GC patients with lung metastasis in this study; however, the reason remains unclear. We speculated that it may be due to the low sample size. Besides, the present study also revealed that patients with single-site lung metastasis exhibited a favorable prognosis following resection of the metastatic lesion. Several retrospective studies reported a survival rate of nearly $30 \%$ at 5-years and improved prognosis for patients with advanced GC who underwent lung metastasis resection [30-32]. However, Sun et al. suggested that the primary resection of GC with lung metastasis had no significant survival benefit [33]. As the lung function of GC patients with lung metastases is poor, and the quality of life may improve after the removal of the diseased lung. Considering these pieces of evidence, we implied that resection of lung metastasis was a reasonable decision. Furthermore, a prospective clinical trial (REGATTA) revealed that chemotherapy combined with surgery was not beneficial to patients with advanced GC and was terminated in advanced cases [34]. In contrast, our study also suggested that chemotherapy combined with primary tumor resection can improve the survival of patients with brain, bone and liver metastasis; apparently, only in the GC patients with lung metastasis, chemotherapy combined with metastasectomy was conducive to survival. Overall, these findings will allow for improved surgical planning strategies for metastatic GC patients. Thus, according to different sites of metastasis, different treatment strategies should be adopted to achieve optimal survival and improve patient's quality of life.

Furthermore, the results of the present study also revealed that the survival outcomes of GC patients with a single-site distant metastasis varied based on the different metastatic sites. Compared to patients with brain and bone metastases, GC patients with lung and liver metastases exhibited better CSS and OS. Moreover, GC patients with single-site metastasis exhibited better CSS and OS than GC patients with multi-organ metastases. Furthermore, surgery of the primary tumor largely improved the prognosis of these patients. The sites of metastases and the number of metastases also influenced the prognosis of metastatic GC patients. Of note, both lung metastasis and liver metastasis are easy to detect with the advancing imaging technologies, and the prognosis of these GC patients is relatively better than for those GC patients with brain metastasis or bone metastasis. These trends were similar to colorectal cancer. However, in pancreatic cancer, no survival difference was observed between patients with single-siteversus multiple site metastases $[35,36]$.

Although this study was carefully conducted, several limitations to this study do exist. First, this was a retrospective study based on the SEER database; therefore, the information relevant to the specific therapy and personal history of patients that could affect prognosis was not available. Second, we included four metastatic sites only as information about metastases to other sites was unavailable, specifically peritoneal metastasis. Peritoneal metastasis plays a crucial role in the diagnosis and treatment of advanced patients with GC, which may cause selection bias. Third, as only less than one third of the patients underwent both primary and metastatic lesion resection, therefore, no single analysis could be performed including only the patients who underwent both primary and metastatic lesion resection in this study. Finally, the database began to release information on GC metastases in 2010 and 
accordingly, the follow-up time in this study was not adequately long enough. Despite these limitations, the present study for the first time was able to comprehensively analyze the effect of primary and metastatic lesion resection on the prognosis of metastatic GC.

\section{Conclusions}

The findings of the present study indicated that the site of metastasis was an independent prognostic factor for metastatic GC. The prognosis of GC patients with liver and lung metastases was better than that of GC patients with bone or brain metastases. Furthermore, outcomes of surgery of primary or metastatic tumors were associated considerably with age, location of the tumor, histological type, grade, site of metastasis, and neoadjuvant chemotherapy. Overall, the curative effect of primary tumor resection on GC patients with liver and lung metastases was favorable for the survival of GC patients with metastases. Surgery had some survival benefits to patients with metastatic GC; however, further studies are warranted on carefully selected patients to validate these findings.

\section{Declarations}

\section{Acknowledgments}

None.

\section{Authors' contributions}

YL contributed to the manuscript preparation and data analysis. YH contributed to the study design. DX contributed to the manuscript editing. $\mathrm{XC}, \mathrm{TH}$, and SL contributed to the image collection. All authors read and approved the final manuscript.

\section{Funding}

This study is supported by the Department of Science \& Technology of Luzhou City (No.2018LZXNYDGP01), and by the Department of Science \& Technology of Liangshan Prefecture (No.18YYJS0104).

\section{Availability of data and materials}

Data available.

\section{Compliance with ethics guidelines}

This study was approved by the Ethics Committee of the Affiliated Hospital of Southwest Medical University, Luzhou City, China.

\section{Consent for publication}

Not applicable. 


\section{Conflict of interest}

The authors declare that they have no conflicts of interest.

\section{References}

1. Bray F, Ferlay J, Soerjomataram I et al. Global cancer statistics 2018: GLOBOCAN estimates of incidence and mortality worldwide for 36 cancers in 185 countries. CA Cancer J Clin 2018, 68(6):394424.

2. Siegel RL, Miller KD, Jemal A. Cancer statistics, 2019. CA Cancer J Clin 2019, 69(1):7-34.

3. Hundahl SA.Staging, Stage Migration, and Patterns of Spreadin Gastric Cancer. Semin Radiat Oncol 2002, 12(2):141-149.

4. Matias Riihimäki AH, Kristina Sundquist, Jan Sundquist et al. Metastatic spread in patients with gastric cancer. Oncotarget 2016, 7(32):52307-52316.

5. Nashimoto A, Akazawa K, Isobe Y et al. Gastric cancer treated in 2002 in Japan: 2009 annual report of the JGCA nationwide registry. Gastric Cancer 2013, 16(1):1-27.

6. Bernards N, Creemers GJ, Nieuwenhuijzen GA et al. No improvement in median survival for patients with metastatic gastric cancer despite increased use of chemotherapy. Ann Oncol 2013, 24(12):30563060 .

7. Qiu JL, Deng MG, Li W et al. Hepatic resection for synchronous hepatic metastasis from gastric cancer. Eur J Surg Oncol 2013, 39(7):694-700.

8. Markar SR, Mackenzie H, Mikhail S et al. Surgical resection of hepatic metastases from gastric cancer: outcomes from national series in England. Gastric Cancer 2017, 20(2):379-386.

9. He MM, Zhang DS, Wang F et al. The role of non-curative surgery in incurable, asymptomatic advanced gastric cancer. PLoS One 2013, 8(12):e83921.

10. Kulig P, Sierzega M, Kowalczyk T et al. Non-curative gastrectomy for metastatic gastric cancer: rationale and long-term outcome in multicenter settings. Eur J Surg Oncol 2012, 38(6):490-496.

11. Ebinger SM, Warschkow R, Tarantino I et al. Modest overall survival improvements from 1998 to 2009 in metastatic gastric cancer patients: a population-based SEER analysis. Gastric Cancer 2016, 19(3):723-734.

12. Chang YR, Han DS, Kong SH et al. The value of palliative gastrectomy in gastric cancer with distant metastasis. Ann Surg Oncol 2012, 19(4):1231-1239.

13. Choi YJ, Kim DH, Han HS et al. Long-term survival after gastrectomy and metastasectomy for gastric cancer with synchronous bone metastasis. World J Gastroenterol 2018, 24(1):150-156.

14. Kojima $\mathrm{H}$, Isaka $\mathrm{M}$, Takahashi $\mathrm{S}$ et al. Long-term survival after pulmonary resections for multiple metastases from gastric cancer: A case report. Int J Surg Case Rep 2018, 45:87-90.

15. Digklia A, Wagner AD. Advanced gastric cancer: Current treatment landscape and future perspectives. World J Gastroenterol 2016, 22(8):2403-2414. 
16. Wagner AD, Syn NL, Moehler M et al. Chemotherapy for advanced gastric cancer. Cochrane Database Syst Rev 2017, 8:CD004064.

17. Daniele Marrelli M, Franco Roviello, MD, Alfonso De Stefano, MD et al. Risk Factors for Liver Metastases After Curative Surgical Procedures for Gastric Cancer: A Prospective Study of 208 Patients Treated with Surgical Resection. American College of Surgeons 2004, 198(1):51-58.

18. Kong JH, Lee J, Yi CA et al. Lung metastases in metastatic gastric cancer: pattern of lung metastases and clinical outcome. Gastric Cancer 2012, 15(3):292-298.

19. KITAOKA KYaH. Bone Metastasis of Gastric Cancer. Jpn J Surg 1983, 13(3):173-176.

20. Yuichi Kasakura MF, Fumiro Mochizuki, Tetsuro Suzuki et al. Clinicopathological Study of Brain Metastasis in Gastric Cancer Patients. Surg Today 2000, 30(6):485-490.

21. Kodera $Y$, Fujitani K, Fukushima $N$ et al. Surgical resection of hepatic metastasis from gastric cancer: a review and new recommendation in the Japanese gastric cancer treatment guidelines. Gastric Cancer 2014, 17(2):206-212.

22. Matias Riihimäki AH, Kristina Sundquist, Jan Sundquist et al. Metastatic spread in patients with gastric cancer. Oncotarget 2016, 7(32):52307-52316.

23. Qiu MZ, Shi SM, Chen ZH et al. Frequency and clinicopathological features of metastasis to liver, lung, bone, and brain from gastric cancer: A SEER-based study. Cancer Med 2018, 7(8):3662-3672.

24. Turkoz FP, Solak M, Kilickap $S$ et al. Bone metastasis from gastric cancer: the incidence, clinicopathological features, and influence on survival. J Gastric Cancer 2014, 14(3):164-172.

25. Liu G, Xu M, Gao T et al. Surgical Compliance and Outcomes in Gastric Cancer: a population-based cohort study. J Cancer 2019, 10(4):779-788.

26. Hass HG, Smith $U$, Jager $C$ et al. Signet ring cell carcinoma of the stomach is significantly associated with poor prognosis and diffuse gastric cancer (Lauren's): single-center experience of 160 cases. Onkologie 2011, 34(12):682-686.

27. Song Z, Wu Y, Yang J et al. Progress in the treatment of advanced gastric cancer. Tumour Biol 2017, 39(7):1010428317714626.

28. Cheon SH, Rha SY, Jeung HC et al. Survival benefit of combined curative resection of the stomach (D2 resection) and liver in gastric cancer patients with liver metastases. Ann Oncol 2008, 19(6):11461153.

29. Sun Z, Liu H, Yu J et al. Frequency and Prognosis of Pulmonary Metastases in Newly Diagnosed Gastric Cancer. Front Oncol 2019, 9:671.

30. Iijima Y, Akiyama H, Atari M et al. Pulmonary Resection for Metastatic Gastric Cancer. Ann Thorac Cardiovasc Surg 2016, 22(4):230-236.

31. Clinton D. Kemp M, Mio Kitano, MD, Sid Kerkar, MD et al. Pulmonary Resection for Metastatic Gastric Cancer. J Thorac Oncol 2010, 5(11):1796-1805.

32. Shiono $\mathrm{S}$, Sato $\mathrm{T}$, Horio $\mathrm{H}$ et al. Outcomes and prognostic factors of survival after pulmonary resection for metastatic gastric cancer. Eur J Cardiothorac Surg 2013, 43(1):e13-16. 
33. Guner A, Yildirim R. Surgical management of metastatic gastric cancer: moving beyond the guidelines. Transl Gastroenterol Hepatol 2019, 4:58.

34. Fujitani K, Yang H-K, Mizusawa J et al. Gastrectomy plus chemotherapy versus chemotherapy alone for advanced gastric cancer with a single non-curable factor (REGATTA): a phase 3, randomised controlled trial. The Lancet Oncology 2016, 17(3):309-318.

35. Luo D, Liu Q, Yu W et al. Prognostic value of distant metastasis sites and surgery in stage IV colorectal cancer: a population-based study. Int J Colorectal Dis 2018, 33(9):1241-1249.

36. Hani Oweira UP, Daniel Helbling, Jan Schmidt et al. Prognostic value of site-specific metastases in pancreatic adenocarcinoma: A Surveillance Epidemiology and End Results database analysis. World J Gastroenterol 2017, 23(10):1872-1880.

\section{Figures}




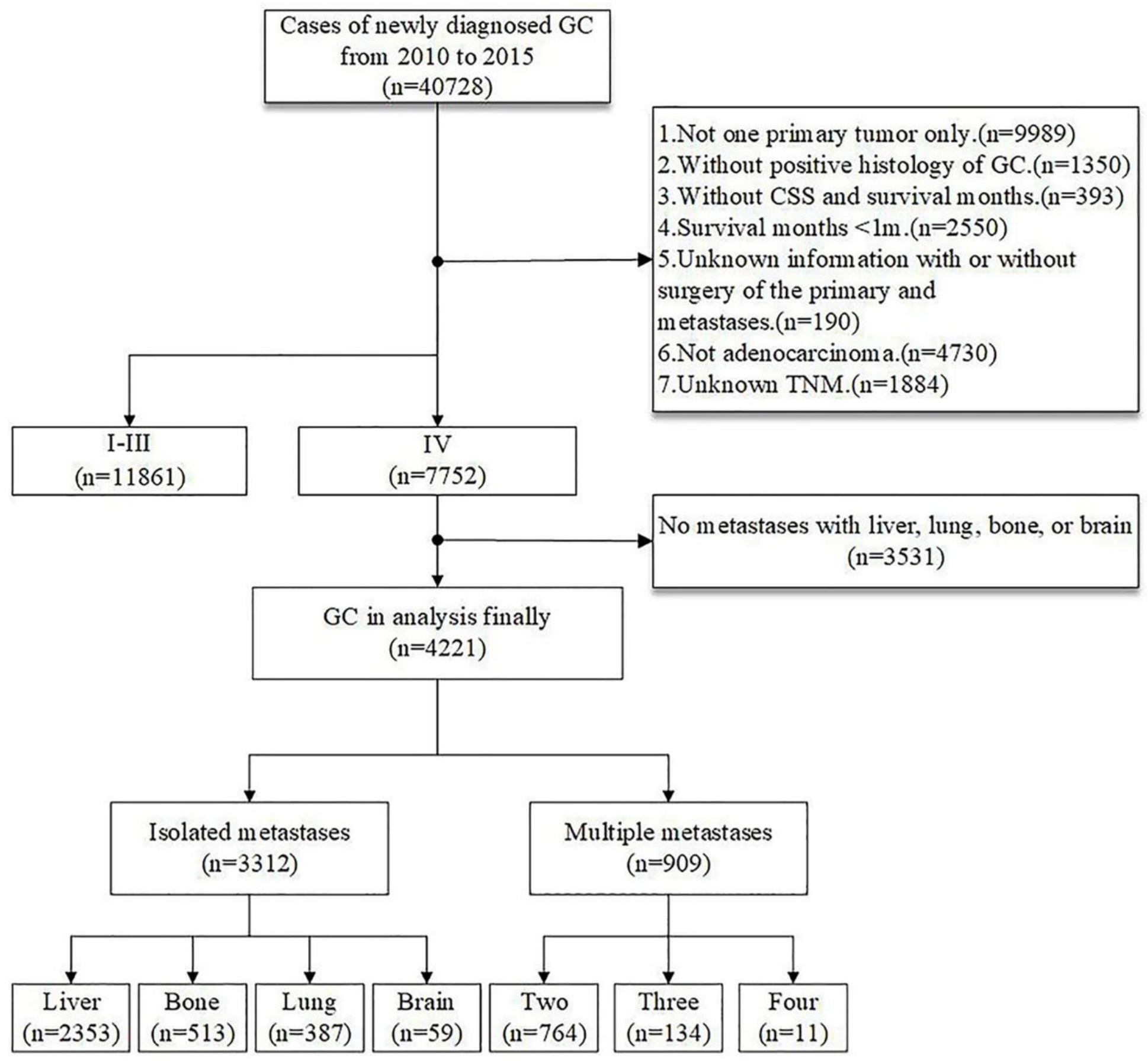

Figure 1

Schematic representation of the data extraction procedure applied in the study 

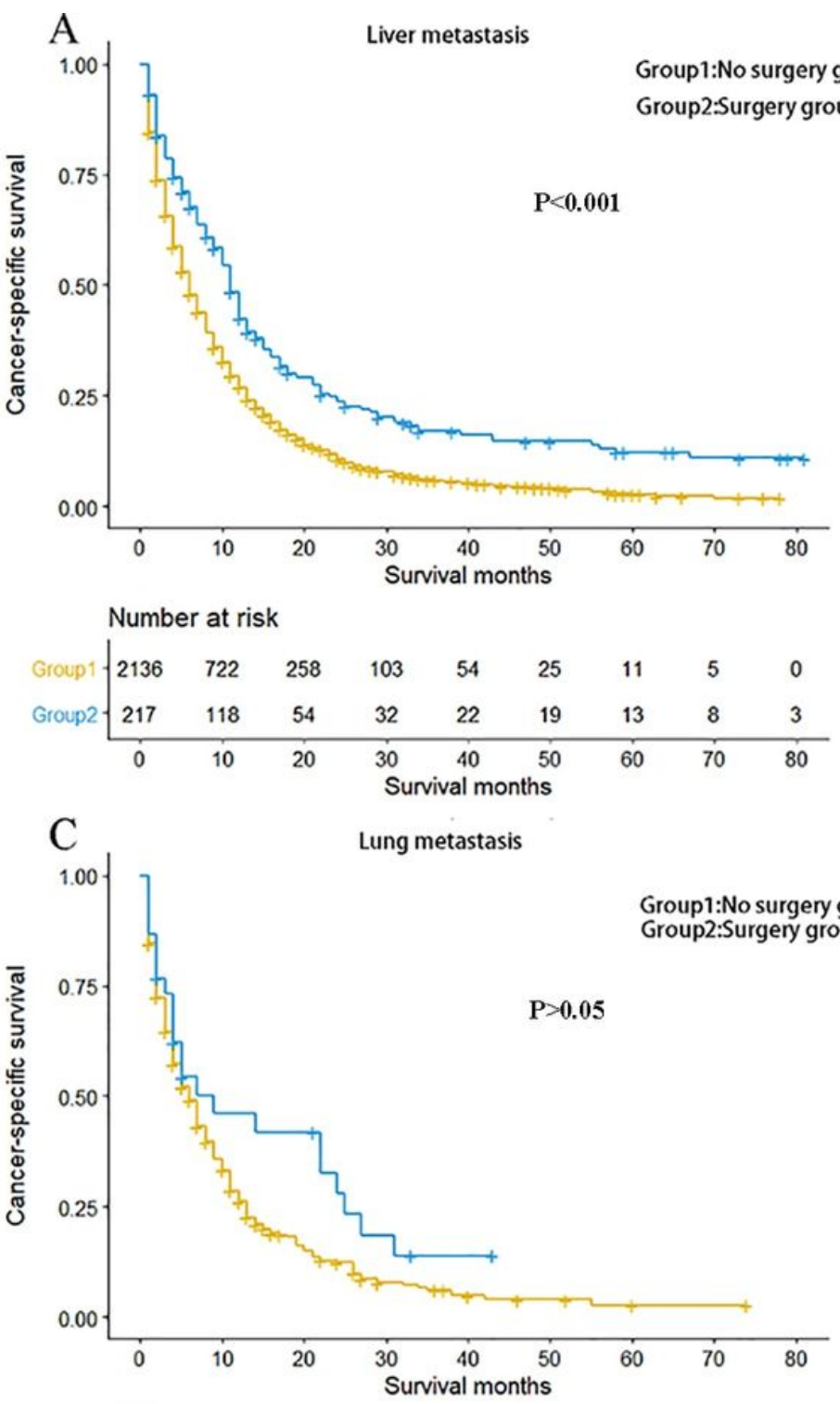

Number at risk

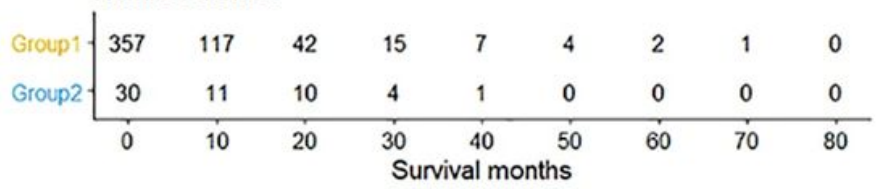

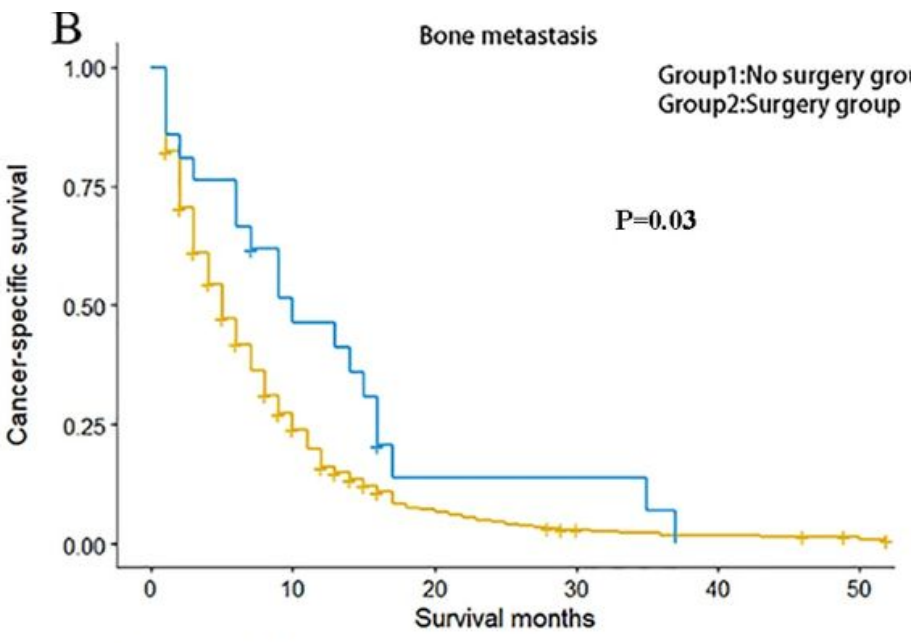
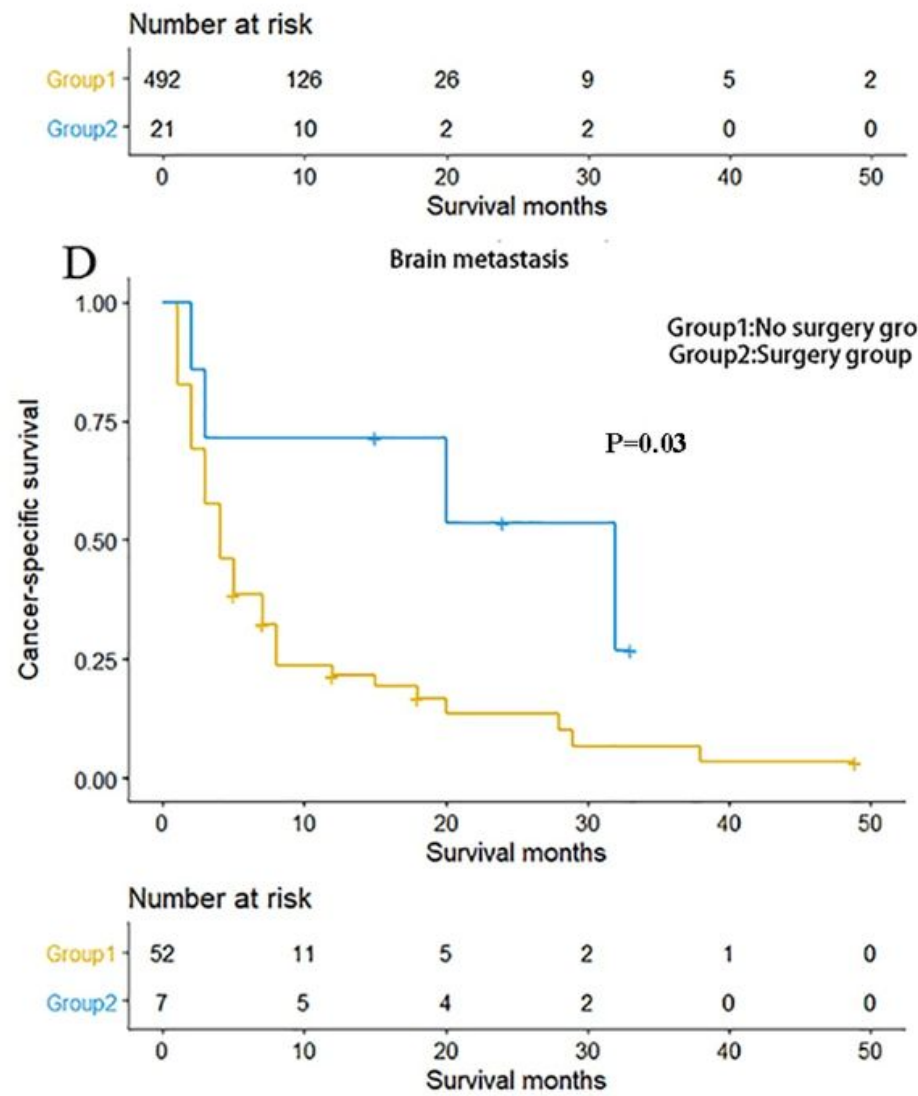

Figure 2

Kaplan-Meier curve of cancer-specific survival based on whether or not surgery of the primary tumor was performed. A Patients with single-site liver metastasis. B Patients with single-site bone metastasis. C Patients with single-site lung metastasis. D Patients with single-site brain metastasis. 

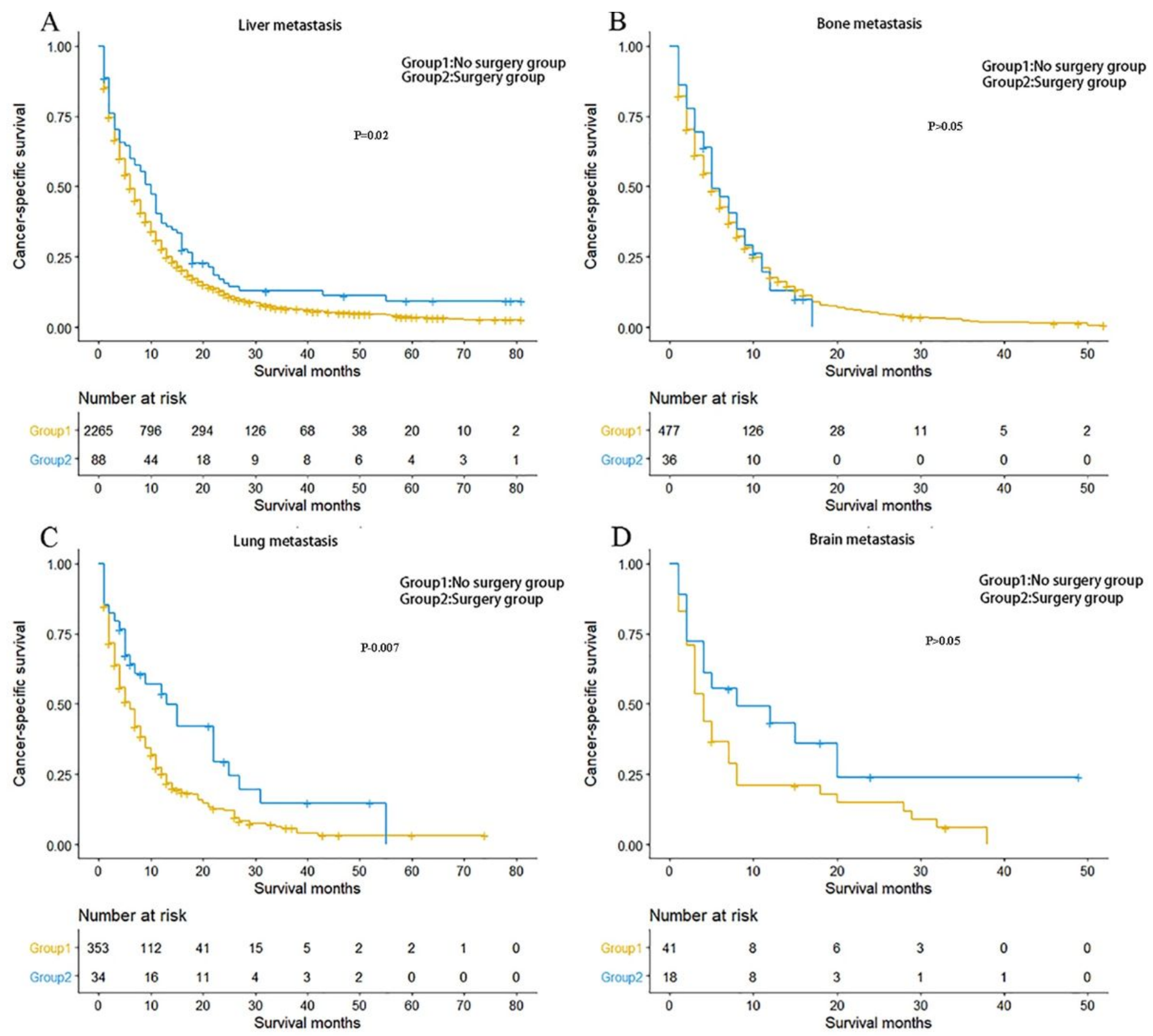

\section{Figure 3}

Kaplan-Meier curve of cancer-specific survival based on whether or not surgery of the metastatic lesion was performed. A Patients with single-site liver metastasis. B Patients with single-site bone metastasis. C Patients with single-site lung metastasis. D Patients with single-site isolated brain metastasis. 

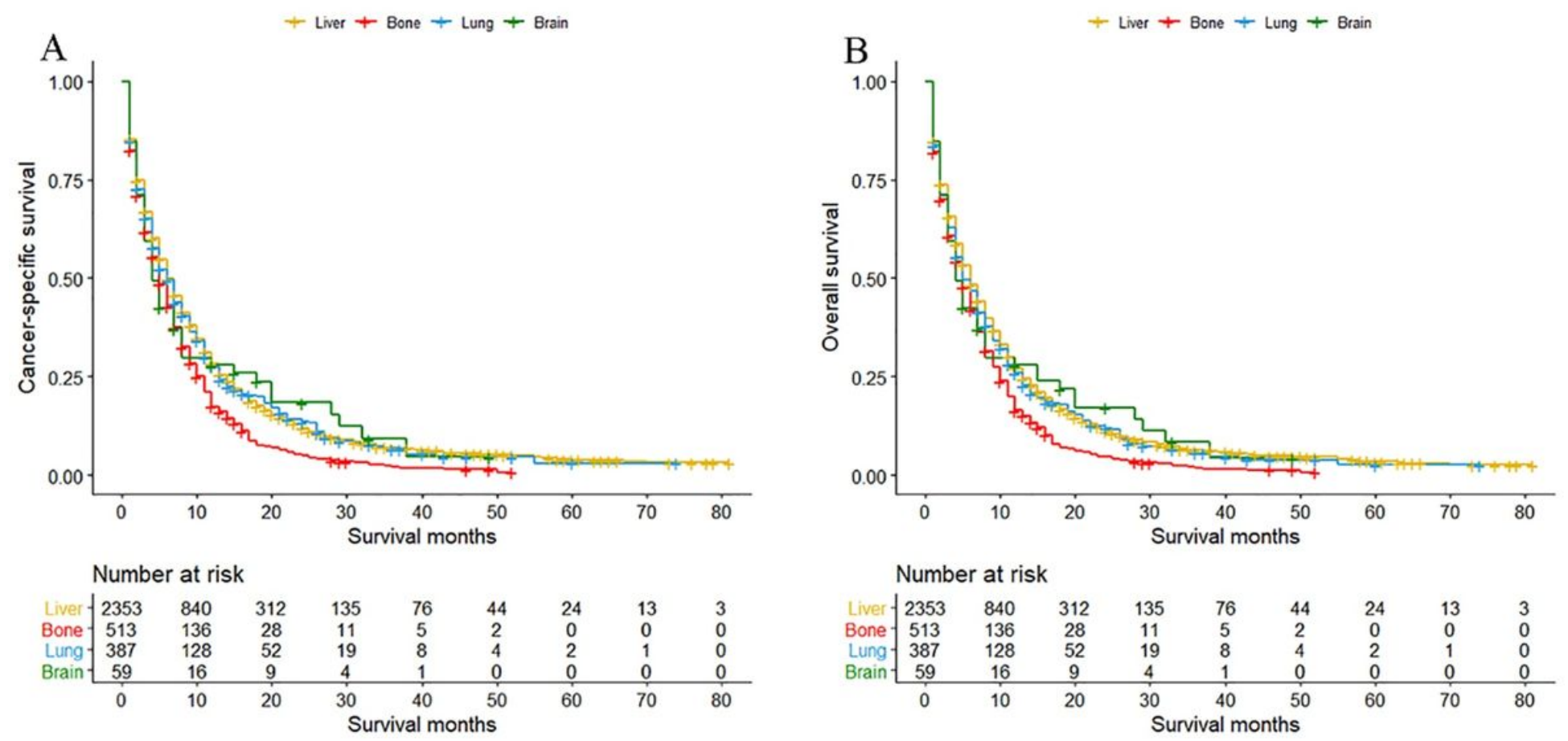

\section{Figure 4}

Kaplan-Meier curve of A cancer-specific survival and B overall survival based on the single-site organ metastases.

\section{Supplementary Files}

This is a list of supplementary files associated with this preprint. Click to download.

- SupplementaryTable.2.docx

- SupplementaryFig.2.tif

- SupplementaryFig.4.tif

- SupplementaryFig.1.tif

- SupplementaryFig.3.tif

- SupplementaryTable.1.docx 